\title{
Undirecting membership in models of Anti-Foundation
}

\author{
Bea Adam-Day(D and Peter J. Cameron(i)
}

\begin{abstract}
It is known that, if we take a countable model of Zermelo-Fraenkel set theory ZFC and "undirect" the membership relation (that is, make a graph by joining $x$ to $y$ if either $x \in y$ or $y \in x$ ), we obtain the Erdös-Rényi random graph. The crucial axiom in the proof of this is the Axiom of Foundation; so it is natural to wonder what happens if we delete this axiom, or replace it by an alternative (such as Aczel's Anti-Foundation Axiom). The resulting graph may fail to be simple; it may have loops (if $x \in x$ for some $x$ ) or multiple edges (if $x \in y$ and $y \in x$ for some distinct $x, y$ ). We show that, in ZFA, if we keep the loops and ignore the multiple edges, we obtain the "random loopy graph" (which is $\aleph_{0}$-categorical and homogeneous), but if we keep multiple edges, the resulting graph is not $\aleph_{0}$-categorical, but has infinitely many 1-types. Moreover, if we keep only loops and double edges and discard single edges, the resulting graph contains countably many connected components isomorphic to any given finite connected graph with loops.
\end{abstract}

Mathematics Subject Classification. Primary 05C63; Secondary 03E99.

Keywords. Erdős-Rényi random graph, Zermelo-Fraenkel axioms, Anti-Foundation Axiom.

\section{Introduction}

According to the downward Löwenheim-Skolem theorem [9, Corollary 3.1.4], if a first-order theory in a countable language is consistent, then it has a countable model. In particular, Zermelo-Fraenkel set theory ZFC, if consistent, has a countable model. (This is the source of the Skolem paradox, since the existence of uncountable sets is a theorem of ZFC.) Indeed there are many different countable models, but they all have a common feature. To describe this, we briefly introduce the Erdős-Rényi random graph (sometimes referred to as Rado's graph, for reasons we will see).

Erdős and Rényi [7] showed that there is a countable graph $R$ such that, if a random graph $X$ on a fixed countable vertex set is chosen by selecting edges independently with probability $1 / 2$ (or, indeed, any fixed $p$ with $0<p<1$ ), then $X$ is isomorphic to $R$ almost surely (that is, with probability 1$)$. Moreover, 
$R$ is highly symmetric; they showed that such a graph has infinitely many automorphisms, but in fact it is homogeneous: any isomorphism between finite induced subgraphs extends to an automorphism. Erdős and Rényi gave a nonconstructive existence proof, based on the following property, called the Alice's Restaurant property, or ARP:

Given any two disjoint finite sets $U$ and $V$ of vertices, there is a vertex $z$ joined to every vertex in $U$ and no vertex in $V$.

In other terminology, $R$ is the Fraïssé limit of the class of finite graphs. For further discussion of the graph $R$, including the theorem below, we refer to [5]; for Fraïssé's theorem, see [9, Theorem 6.1.2].

A model of Zermelo-Fraenkel set theory ZF consists of a collection of objects called sets, and a binary relation $\in$ on this collection. In other words, it is a directed graph. By "undirecting" this relation $\in$, that is, defining an undirected graph in which $x$ and $y$ are adjacent if either $x \in y$ or $y \in x$, we obtain a simple graph. (At the end of this section, we state the Axiom of Foundation, one of the axioms of $\mathrm{ZF}$, and show that it forbids directed cycles for the membership relation; in particular it forbids loops $(x \in x)$ and double edges $(x \in y \in x)$ in the undirected graph.) We call this the membership graph of the model.

Theorem 1. The membership graph of a countable model of ZFC is isomorphic to the Erdös-Rényi random graph $R$.

Proof. We verify the ARP. Let $U$ and $V$ be finite disjoint sets of vertices. Take $z=U \cup\{V\}$. (The existence of $z$ is guaranteed by the Pairing and Union axioms.) If $u \in U$, then $u \in z$, so $z$ is joined to $u$. Suppose, for a contradiction, that $z$ is joined to a vertex $v \in V$. There are two cases:

- $v \in z$. Since $v \notin U$, we must have $v=V$, so $v \in v$, contradicting Foundation.

- $z \in v$. Then $v \in V \in z \in v$, also contradicting Foundation.

Remark. Observe that, in the proof, we use only the Empty Set axiom (asserting that sets exist), the axioms of Pairing and Union, and the Axiom of Foundation. The other axioms (Infinity, Selection, Choice, and so on) are not required.

In particular, there is a standard model of $\mathrm{ZFC}$ with the negation of the Axiom of Infinity, or hereditarily finite set theory HF. We take the sets to be the natural numbers, and represent a finite set $\left\{a_{1}, \ldots, a_{n}\right\}$ of natural numbers by $b=2^{a_{1}}+\cdots+2^{a_{n}}$, so that, for $a<b$, we have $a$ joined to $b$ if and only if the $a$ th digit in the base 2 expansion of $b$ is 1 . The model of $R$ given by undirecting this membership relation is precisely the graph constructed by Rado [10].

We are grateful to the referee for the observation that the argument also shows the following: if the Continuum Hypothesis holds, then the undirected 
membership relation on the hereditarily countable sets is a universal graph of cardinality $\aleph_{1}$.

The Axiom of Foundation We state the Axiom of Foundation, and its role in forbidding directed cycles for the membership relation in models of ZF; see $[4,6]$ for more details.

The Axiom of Foundation states:

For every non-empty set $x$, there exists $y \in x$ such that $x \cap y=\emptyset$.

Suppose that there were a directed cycle $x_{0} \in x_{1} \in \cdots \in x_{n-1} \in x_{0}$. Let $x=\left\{x_{0}, x_{1}, \ldots, x_{n-1}\right\}$. For any $y \in x$, say $y=x_{i}$, we have $x_{i-1} \in x \cap y$, contradicting the Axiom of Foundation.

In fact the axiom also forbids infinite descending chains for the membership relation, and is "equivalent" to this (but not by a first-order implication since there is no first-order formula forbidding such chains).

\section{The Anti-Foundation Axiom}

Since the Axiom of Foundation is required for the proof of Theorem 1, what happens if we delete it, or replace it by an alternative? We consider this question when Foundation is replaced by Aczel's Anti-Foundation Axiom [1]. Following Barwise and Moss [3], we use this axiom in the form of the Solution Lemma [3, p.72], which we briefly discuss. Other formulations of the axiom exist, see for example [8], but this form suits our purpose.

Let $X$ be a set of "indeterminates", and $A$ a set of sets called "atoms". A flat system of equations is a set of equations of the form $x=S_{x}$, where $S_{x}$ is a subset of $X \cup A$ for each $x \in X$. A solution to the system is an assignment of sets to the indeterminates so that the equations become true.

For example, if $A=\{a, b\}$ with $a$ and $b$ distinct, then

$$
\begin{aligned}
& x=\{y, a\}, \\
& y=\{x, b\}
\end{aligned}
$$

is a flat system of equations.

The Anti-Foundation Axiom, or AFA, asserts that any flat system of equations has a unique solution. (In fact, the proofs we give here will not require such uniqueness, and so all the results hold for a weaker form of AntiFoundation.)

Note that the solution to the above system will satisfy $x \in y, y \in x$ and $x \neq y$ so will correspond to a double edge in the membership graph. Similarly, the solution to $x=\{x\}$ will give a loop in the graph.

The axioms system ZFA denotes ZFC with the Axiom of Foundation deleted and replaced by the axiom AFA. Our concern is with membership graphs of 
models of ZFA. We note in passing that, if ZFC is consistent then so is ZFA: see [3, Chapter 9].

Note that Barwise and Moss work in a set theory containing "urelements" which are not themselves sets; this makes no difference to our arguments.

\section{Membership graphs of models of ZFA}

The argument showing that the membership graph of a model of ZFC is a simple graph does depend on Foundation, as we saw. In ZFA, we have sets $x$ with $x \in x$, giving loops in the graph; and pairs $x, y$ of distinct sets with $x \in y \in x$, giving double edges.

The random loopy graph is obtained with probability 1 if we choose a graph on a countable vertex set by choosing edges (including loop edges) from pairs of not necessarily distinct vertices independently with probability $1 / 2$. It is homogeneous, and is the Fraïssé limit of the class of finite loopy graphs. The relevant version of the Alice's Restaurant property characterises it as the countable graph such that, for any two finite disjoint sets $U$ and $V$ of vertices, there are vertices $z_{1}$ and $z_{2}$, where $z_{1}$ is loopless and $z_{2}$ has a loop, each joined to all vertices in $U$ and to none in $V$. The proof is very similar to the usual proof for the random graph, and we will not give it here.

Theorem 2. The membership graph of a countable model of ZFA, ignoring multiple edges but keeping loops, is isomorphic to the random loopy graph.

Proof. We begin with some preliminaries. In a model of a subset of ZF including at least Selection, there is no set whose members are all sets. For, if such a set $S$ exists, then Selection would give Bertrand Russell's set $R=\{x \in S$ : $x \notin x\}$, whose existence leads to a contraction on examining whether $R \in R$ or not.

It follows that, if the Union axiom also holds, there is no set $S^{\prime}$ which contains all the $p$-element sets, for a fixed positive natural number $p$ : for the union of $S^{\prime}$ would be $S$. In particular, if $T$ is any set, then there is a set of cardinality $p$ which is not a member of $T$.

Now let $\Gamma$ be the membership graph of a countable model of ZFA, with loops but no double edges. We will show that $\Gamma$ satisfies the loopy version of ARP. For this let $\left\{u_{1}, \ldots, u_{m}\right\}$ and $\left\{v_{1}, \ldots, v_{n}\right\}$ be disjoint sets, and let $z_{1}=\left\{x, u_{1}, \ldots, u_{m}\right\}$ and $z_{2}=\left\{z_{2}, x, u_{1}, \ldots, u_{m}\right\}$, where $x$ is a vertex satisfying the following conditions:

- $x$ is not equal to any of the $v_{j}$;

- $x$ is not contained in any of the $v_{j}$ or the $u_{i}$;

- $x$ is not contained in any of the sets contained in any of the $v_{j}$.

Such an $x$ exists, since otherwise the union of $V, \cup U, \cup V$, and $\cup U V$ would contain every set, a contradiction. 
Furthermore, we may assume that $|x|=m+3$, since by our earlier remarks there is a set of this cardinality not a member of the "forbidden set" $V U$ $(\bigcup U) \cup(\bigcup V) \cup(\bigcup \cup V)$ above.

We remark that the existence of $z_{1}$ follows simply from Pairing and Union; for $z_{2}$, we invoke Anti-Foundation, letting $z_{2}$ be the unique solution of the equation

$$
z=\left\{z, x, u_{1}, \ldots, u_{m}\right\} .
$$

Both $z_{1}$ and $z_{2}$ are joined to all the vertices $u_{i}$; and by construction, there is a loop on $z_{2}$. We claim that there is no loop on $z_{1}$. For such a loop would imply one of the following:

- $z_{1}=u_{i}$ for some $i$. Then we have $x \in u_{i}$, contradicting our choice of $x$.

- $z_{1}=x$. But we chose $x$ with $|x|=m+3$, whereas $\left|z_{1}\right| \leq m+1$. (Note that in the same way we see that $z_{2} \neq x$.)

Finally we have to show that $z_{1}$ and $z_{2}$ are not joined to any $v_{j}$. We cannot have any $v_{j}$ contained in $z_{1}$ or $z_{2}$; for the $v_{j}$ are distinct from the $u_{i}$ by hypothesis, not equal to $x$ by choice of $x$, and not equal to $z_{2}$ since if so then $x$ would be a member of $v_{j}$, again contrary to our choice of $x$. Also we cannot have $z_{1}$ or $z_{2} \in v_{j}$, since if so then $x$ belongs to a member of $v_{j}$, again contrary to our choice of $x$.

Remark. As in the case of ZFC, it is interesting to note which axioms are actually used in the proof. The Empty Set, Pairing and Union axioms are once again used; of course, the Anti-Foundation Axiom is used; and as well, we use the Selection Axiom.

What happens if we keep the multiple edges? We cannot describe all graphs that can arise, but we note that there is no such graph which has the nice properties of $\aleph_{0}$-categoricity and homogeneity which hold in the random and random loopy graphs. This will follow from the theorem of Engeler, RyllNardzewski and Svenonius (see [9, Theorem 6.3.1]), according to which a countable first-order structure is $\aleph_{0}$-categorical (that is, determined uniquely by its first-order theory and the property of countability) if and only if its theory has only finitely many $n$-types for all $n$. An $n$-type of a first order theory $T$ is a set $\Phi(\bar{x})$ of formulas with at most $n$ free variables in the language of $T$ such that $T \cup\{\exists \bar{x} \wedge \phi\}$ is consistent for every finite subset $\phi(\bar{x})$ of $\Phi$. In a sense, an $n$-type describes how a set of up to $n$ elements might behave in a model of $T$.

Theorem 3. The membership graph of a countable model of ZFA, keeping double edges, is not $\aleph_{0}$-categorical.

Proof. Take a countable model of ZFA. Let $a_{n}$ be distinct well-founded sets for $n \in \mathbb{N}$, for example, the natural numbers. For every natural number $n$, consider the equations

$$
y=\left\{x_{0}, \ldots, x_{n-1}\right\},
$$




$$
x_{i}=\left\{y, a_{i}\right\} \text { for } i=0, \ldots, n-1 .
$$

By AFA, these equations have a unique solution in the model. We have $x_{i} \in y$ and $y \in x_{i}$, so all the edges $\left\{x_{i}, y\right\}$ are double. (These sets are all distinct, by extension.) There are no further double edges on $y$, since if $\{y, z\}$ is a double edge then $z \in y$ and so $z=x_{i}$ for some $i$.

Thus, for every natural number $n$, there is a set lying on exactly $n$ double edges. Since this property of the set is expressible in first-order logic there are infinitely many 1 -types in the graph, and so the graph cannot be $\aleph_{0}$-categorical, by the theorem of Engeler, Ryll-Nardzewski and Svenonius.

Moreover, we can take the infinite set of equations

$$
\begin{aligned}
y & =\left\{x_{n}: n \in \mathbb{N}\right\}, \\
x_{n} & =\left\{y, a_{n}\right\} \text { for } n \in \mathbb{N} .
\end{aligned}
$$

A solution to these equations will be a point lying on infinitely many double edges.

Another natural reduct is obtained by keeping only the double edges. The double-edge graph of a model of ZFA has as vertices the sets and as edges all pairs $\{x, y\}$ with $x \in y$ and $y \in x$ (allowing $x=y$ ). Thus, it includes loops and double edges but omits all "conventional" instances of the membership relation (where $a \in b$ but $b \notin a$ ).

Theorem 4. Let $D$ be the double-edge graph of a countable model of ZFA. Then, for any finite connected loopy graph $\Gamma, D$ has infinitely many connected components isomorphic to $\Gamma$. It also has at least one infinite component.

Proof. An example will illustrate the general proof. Let $\Gamma$ be the 4 -cycle with edges $\left\{v_{0}, v_{1}\right\},\left\{v_{1}, v_{2}\right\},\left\{v_{2}, v_{3}\right\}$ and $\left\{v_{3}, v_{0}\right\}$, together with a loop at $v_{0}$. Take any four well-founded sets $a_{0}, a_{1}, a_{2}, a_{3}$ (for example, the first four natural numbers), and consider the equations

$$
\begin{aligned}
& y_{0}=\left\{a_{0}, y_{0}, y_{1}, y_{3}\right\}, \\
& y_{1}=\left\{a_{1}, y_{0}, y_{2}\right\}, \\
& y_{2}=\left\{a_{2}, y_{1}, y_{3}\right\}, \\
& y_{3}=\left\{a_{3}, y_{0}, y_{2}\right\} .
\end{aligned}
$$

The unique solution gives an induced subgraph isomorphic to $\Gamma$. Note that there are no other double edges meeting these vertices: if, say, $\left\{y_{1}, x\right\}$ were a double edge, then $x \in y_{1}$, and so by Extensionality, $x=a_{1}$ or $x=y_{0}$ or $x=y_{2}$; the first is impossible since $y_{1} \notin a_{1}$ by assumption. So the given set is a connected component.

Since there are infinitely many possible choices of $a_{0}, \ldots, a_{3}$, there are infinitely many such connected components.

We saw earlier that there is a vertex with infinite valency; it lies in an infinite component of $D$. 
This leaves a few questions which we have not been able to answer in this current work.

1. Is it true that the first-order theory of the membership graph of a countable model of ZFA has infinitely many countable models?

2. Is it true that there are infinitely many non-isomorphic graphs which are membership graphs of countable models of ZFA?

3. Can more be said about infinite connected components of the double-edge graph?

4. What about models of ZFA where the Axiom of Infinity is replaced with its negation?

5. Is it true that, if two countable multigraphs are elementarily equivalent, and one is the membership graph of a model of ZFA, then so is the other?

However, in a sequel by Adam-Day et al. [2] the first two questions are answered affirmatively and a characterisation of the connected components of double-edge graphs is given, thus answering the third question. The analogue to Question 5 for double-edge graphs is shown to be negative, and indeed it is shown that, for any double-edge graph, there is an elementarily equivalent countable structure which is not itself a double-edge graph.

Open Access. This article is licensed under a Creative Commons Attribution 4.0 International License, which permits use, sharing, adaptation, distribution and reproduction in any medium or format, as long as you give appropriate credit to the original author(s) and the source, provide a link to the Creative Commons licence, and indicate if changes were made. The images or other third party material in this article are included in the article's Creative Commons licence, unless indicated otherwise in a credit line to the material. If material is not included in the article's Creative Commons licence and your intended use is not permitted by statutory regulation or exceeds the permitted use, you will need to obtain permission directly from the copyright holder. To view a copy of this licence, visit http:// creativecommons.org/licenses/by/4.0/.

Publisher's Note Springer Nature remains neutral with regard to jurisdictional claims in published maps and institutional affiliations.

\section{References}

[1] Aczel, P.: Non-Wellfounded Sets. CSLI Publications, Stanford (1988)

[2] Adam-Day, B., Howe, J., Mennuni, R.: On double-membership graphs of models of Anti Foundation. preprint. https://arxiv.org/abs/1908.02708 (2019)

[3] Barwise, J., Moss, L.: Vicious Circles. CSLI Publications, Stanford (1996)

[4] Cameron, P.J.: Sets, Logic and Categories. Springer, London (1999)

[5] Cameron, P.J.: The random graph. In: Graham, R.L., Nešetřil, J., Butler, S. (eds.) The Mathematics of Paul Erdős, vol. II, 2nd edn, pp. 353-378. Springer, New York (2013)

[6] Devlin, K.J.: The Joy of Sets: Foundations of Contemporary Set Theory, 2nd edn. Springer, New York (1991)

[7] Erdős, P., Rényi, A.: Asymmetric graphs. Acta Math. Acad. Sci. Hungar. 14, 295-315 (1963) 
[8] Forti, M., Honsell, F.: Set theory with free construction principles. Annali della Scuola Normale Superiore di Pisa — Classe di Scienze 10, 493-522 (1983)

[9] Hodges, W.A.: A Shorter Model Theory. Cambridge Univesity Press, Cambridge (1997)

[10] Rado, R.: Universal graphs and universal functions. Acta Arith. 9, 331-340 (1964)

\section{Bea Adam-Day}

School of Mathematics

University of Leeds

Woodhouse, Leeds LS2 9JT

UK

e-mail: B.Adam-Day@leeds.ac.uk

Peter J. Cameron

School of Mathematics and Statistics

University of St Andrews

St Andrews, Fife KY16 9SS

UK

e-mail: pjc20@st-andrews.ac.uk

Received: March 26, 2020

Revised: November 3, 2020

Accepted: November 5, 2020 